\title{
ADAPTIVE HYBRID FORCE/POSITION CONTROL OF A FLEXIBLE MANIPULATOR FOR AUTOMATED DEBURRING WITH ON-LINE CUTTING TRAJECTORY MODIFICATION
}

\author{
I-Ching Lin $^{1}$ and Li-Chen Fu ${ }^{1,2}$
}

\author{
Department of Electrical Engineering ${ }^{1}$ \\ Department of Computer Science and Information Engineering ${ }^{2}$ \\ National Taiwan University \\ Taipei, Taiwan, Republic of China
}

\begin{abstract}
In this paper, we propose a method of controlling a deburring flexible manipulators. All of the analysis in the paper are under the curved coordinates. The goal of the automated deburring is to remove the burrs at a constant tangential cutting force. Therefore, maintaining a constant contact normal force and constant tangential cutting force is required. The dynamics of both the deburring process and the flexible manipulator will be investigated in detail, and the latter will be derived using Lagragian method with assume-mode approach. To facilitate the controller design, a singular perturbation technique is then utilized to separate the system into a slow subsystem and a fast subsystem. For solving the deburring problem, a new hybrid force/position controller is proposed for the slow subsystem, it is implemented by adaptive control strategy, whereas a dynamic feedback controller is developed for the fast subsystem. It is shown that both the position tracking error and the force error will converge to zero as time approach infinity. Finally, the computer simulations and experiments of a two-link flexible manipulator confirm the effectiveness of the proposed adaptive controller.
\end{abstract}

\section{INTRODUCTION}

For a variety of reasons, such as to assemble parts properly, prevent injury to workers, the burrs on the part's edge must be removed. However, the manual deburring process is a costly and time-consumming operation, for some machined parts, the cost of the deburring process can even be as high as 35 percent of the total part's cost, result in applying automated deburring system to replace manual opcration to become more and more important. Therefore, the system will be investigated thoroughly in the paper.

To reduce structural vibration to ease the controller design, a robot manipulator is often made rigid and with heavy structure, but several disadvantages may, however, arise such as high-power consumption, low motion speed and low ratio of the payload capacity to the robot weight. Besides, the uncertainties in the manipulator system, the positional accuracy of the endeffector is generally poor, if both the robot manipulator and the part are mostly rigid, the impact from the manipulator to the part will frequently cause damage to each other. Due to these shortcomings of applying conventional rigid robot manipulators, the research on controlling the flexible manipulators has attracted more and more attention nowadays.

There are more and more advance control schemes had been applied for automated deburring, such as impedance control, hybrid force control, teaching and learning control and adaptive control [5][6][7] [10][23][24]. In [2], a precision deburring model has been proposed, it has shown that the relationship between the material removal rate and the cutting force, and point out the tangential cutting force is more sensitive to the variation of burrs than normal cutting force, the control strategy guarantee burr removal while compensating for robot oscillations and small uncertainties in the location of the part relative to the robot and maintain constant normal force and tangential force by the impedance control. An alternative approach is to control the chamfer depth with the minimal depth surface by minimizing the normal cutting force, the control laws are designed based on models combining a deterministic plant with a stochastic disturbance which are identified from experimental data[6]. Since to construct a precise model is very difficult, this fact results in that a deburring scheme based on a human-skill model was proposed [5]. Furthermore, a neural network controller which is equipped with the ability of refining the deburring behavior by learning from the skillful engineers was also developed [7]. Due to the parameters of a robot system may be very difficult to obtain or to measure, and the load grasped by the robot manipulator is usually subject to uncertainty, adaptive con- 
trol schemes have been adopted for solving this kind of uncertainty problem. An adaptive inertia-related controller for tracking problems to improve the earlier adaptive computed-torque controller [19]. A hybrid position/force controller can be used for tracking the desired position and force trajectories simultaneously [17] [10]. The basic concept of the controller is that the task space can be defined with respect to the constraint coordinate and be further decomposed into a position subspace and a force subspace. An alternative approach of the above is to achieve position/force control after applying some state reduction process [14].

In the area of flexible manipulator, there also have been many studies about how to develop satisfactory controllers for flexible manipulators. The closed-form dynamic model of multilink flexible manipulators are completely derived by Luca and Siciliano [12] and, a singular perturbation approach to devise a control for flexible manipulators is proposed by Siciliano and Book [15]. So far, many nonlinear control schemes have been developed for multilink flexible manipulators in the free space [21]. In constraint case, Matsuno et al. [22] have proposed a hybrid position/force controller based on the quasi-static equations. Lian et al. [1] proposed an adaptive hybrid position/force controller based on singular perturbation theory for flexible manipulators in Cartesian space.

In this paper, a deburring process model is presented, and a reduced model in curved coordinates is derived due to the constraints imposed on the end effector. Base on sigular perturbation theory, an adaptive hybrid force/position controller is designed for the slow subsystem, and a dynam:c feedback controller is designed for the fast subsystem. In our Advanced Control Laboratory (ACL) at National Taiwan University (NTU), a 2-link planar flexible manipulator equipped with a grinding tool has been built to demonstrate the performance of the proposed controller.

This paper is organized as follows : Section 2 present the model of the deburring process and the dynamic model in curved coordinates of a constrained flexible manipulator. Further by applying the singular perturbation technique, the original system is decomposed into a slow subsystem and a fast subsystem. In Section 3, an adaptive hybrid force/position controller and a dynamic feedback controller are developed based on the formulation of the two subsystems derived previously. In Section 4, the simulation result and the experimental result will demonstrate the real controlled performance. Finally, some conclusions will be given in Section 5 .

\section{PROBLEM FORMULATION}

\section{Deburring Model}

The objective of deburring is to remove the burrs on the part's edge to get the smoother surface. Hence, the specifications of the part's deburring, in general, are that the force of tangential direction must keep constant to avoid damaging the part and tool, and the

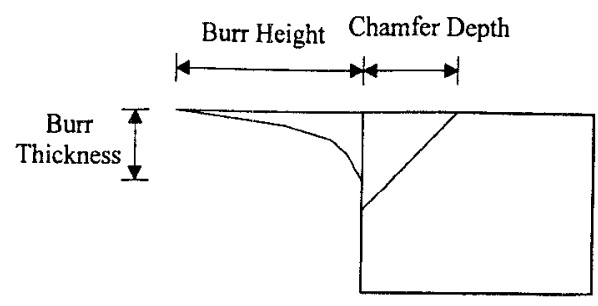

Figure 1: The burr cross-section

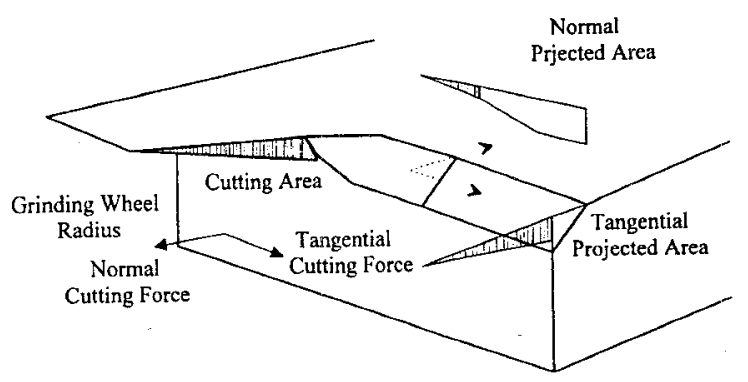

Figure 2: The 3D geometric model of the cutting surface

chamfer depth along the edge should be kept within a given set of tolerences.

According [2], we will introduce the model of the deburring process in the following. Fig. 1 shows the cross-section of a burr, from which burr height, burr thickness, and the chamfer depth are clearly illustrated. We assume that the average burr height is much greater than the average burr thickness and the chamfer depth is usually chosen to be greater than the maximum of the possible burr thickness[2].

According to the above assumption and Fig.2, a three-dimentional geometric model of a cutting surface. Let the cutting area be projected into the plane tangential to the constraint surface and another plane with a normal tangent to the same surface, respectively. The normal projected area of the burr relative to the normal projection of the contact area can be very insignificant, but the tangential projected area of the burr relative to the tangential projection of the contact area becomes much more substantial. Hence, the variation of the tangential projected area with the burr size is much greater than that of the normal projected area with burr size. Because the cutting force is proportional to the contact area, it is sure that the tangential force varies much more significantly with the burr size, but the normal force maintains almost constant magnitude regardless of the burr size. Therefore, a consistent chamfer depth can be obtained by controlling only the normal force.

So far as we know about the tangential cutting force is that it depends on the burr size. In the following, we will derive the tangential cutting force in detail. According to [2], when the grinding tool moves along the edge for deburring, the material removal rate $M R R$ 
can be expressed as:

$$
M R R=\left(A_{c}+A_{b}\right) \times v_{t}
$$

where $A_{c}$ and $A_{b}$ denote the cross sectional area of the chamfer and of the burr, respectively, and $v_{t}$ is the velocity of the grinding tool along the part's edge. In [5], the following model based on a metal cutting theory is introduced.

$$
f_{t}=b_{1}\left|\dot{r}_{t}\right|+b_{2}\left|\dot{r}_{n}\right|+b_{3}
$$

where $\dot{r}_{t}$ is the velocity tangential to the part, $\dot{r}_{n}$ is the velocity normal to the part. For this process model, the vector of process parameters can be written as $b^{T}=$ $\left[b_{1} b_{2} b_{3}\right]$, where $b_{1}=\lambda f(h) / w R, b_{2}=-\lambda g(h) / w R$, and $b_{3}=P_{t h} / w R, f(h)$ and $g(h)$ are functions of burr height $h, P_{t h}$ is the threshold power, $w$, and $R$ are the spindle speed of the grinding tool, and the radius of the grinding wheel, respectively.

In [25], it indicated that $b_{1}$ is proprotional to the cross-sectional area of a burr along the tool's travel direction, $b_{2}$ is negligibally small, and $b_{3}$ is almost constant for the same workpiece material. For the same workpiece material, burr size parameter $b_{1}$ is the main factor for a human worker to determine tool's feedrate for deburring. As a result, we can rewrite the tangential cutting force as follows:

$$
f_{t} \cong b_{1}\left|\dot{r}_{t}\right|+b_{3}
$$

By simplifying the model of the tangential cutting force, a disired positional profile command $r_{t c}$ during the sample-period $T$ can be made, expressed as follows

$r_{t c}(k T)= \begin{cases}r_{t}(k T)+\frac{\dot{r}_{t}(k T)\left(f_{t d}-b_{3}\right)}{f_{t}-b_{3}} T & , \text { if } f_{t}-b_{3}>v_{\epsilon} \\ r_{t}(k T)+v_{0} T & , \text { otherwise }\end{cases}$

where $f_{t d}$ is the desired constant tangential cutting force, $r_{t}$ is the cutting tool position, $k=1,2, \ldots, v_{0}$ is the default feedrate, set to prevent $x$ from changing too fast to damage the part as $\left(f_{t}-b_{3}\right)$ becomes too small, and $v_{\epsilon}>0$ is its offset. When $\dot{r}_{t} \rightarrow \frac{r_{t c}(k T)-r_{t c}((k-1) T)}{T}$, we can get a result that $f_{t}$ closely approximates $f_{t d}$. A continuous desired position trajectory $r_{t d}(t)$ can then be obtained by passing $r_{t c}(k T)$ through a modified first-order hold filter with an initial value $r_{t d}(0)=$ $r_{t c}(0)$ as follows :

$$
r_{t d}(t)=r_{t d}(k T)+\frac{r_{t c}(k T)-r_{t d}((k-1) T)}{T}(t-k T)
$$

Finally, the desired feedrate in the tangential direction has been determined. It should be realized that one can use position control in the direction tangential to the constraint surface and force control in the direction normal to the same surface in order to accomplish the above-mentioned control task in the deburring process.

\section{Dynamic Model of a Deburring Flexible Manipulator in Curved Coordinates}

After having the basic knowledge discussed in the previous section, the dynamic model of the deburring flex- ible manipulator in curved coordinates will be derived in the next.

The dynamic model of a flexible manipulator which moves along the part's edge for deburring can be expressed as

$$
M(q) \ddot{q}+C(q, \dot{q}) \dot{q}+K q+G(q)=\tau+A^{T} \lambda+\tau_{t}
$$

where $\tau_{t} \in R^{N}$ denotes the tangential cutting force in joint-space coordinates, and $\lambda \in R^{m}$ is the vector of Lagrange multipliers [14] associated with the geometric constraints $\Phi^{\prime}(q)=0, A=\frac{\partial \Phi^{\prime}(q)}{\partial q}, \Phi: R^{N} \rightarrow R^{m}$. For convenience, we partition the above dynamic model into the following form:

$$
\begin{array}{r}
{\left[\begin{array}{ll}
M_{r r} & M_{r f} \\
M_{f r} & M_{f f}
\end{array}\right]\left[\begin{array}{c}
\ddot{q}_{r} \\
\ddot{q}_{f}
\end{array}\right]+\left[\begin{array}{ll}
C_{r r} & C_{r f} \\
C_{f r} & C_{f f}
\end{array}\right]\left[\begin{array}{c}
\dot{q}_{r} \\
\dot{q}_{f}
\end{array}\right]} \\
+\left[\begin{array}{c}
G_{1} \\
G_{2}+K q_{f}
\end{array}\right]=\left[\begin{array}{c}
\tau+A_{1}^{T} \lambda+\tau_{t r} \\
A_{2}^{T} \lambda+\tau_{t f}
\end{array}\right]
\end{array}
$$

where $q_{r} \in R^{n},(n \leq 6)$ and $q_{f} \in R^{n_{f}}, n+n_{f}=N$, and $A_{1}=\frac{\partial \Phi}{\partial q_{r}}, A_{2}=\frac{\partial \Phi}{\partial q_{f}}$. The subscript $r$ and $f$ denote the rigid mode part and flexible mode part, respectively.

In order to transform the equations of motion with respect to joint coordinates $q$ into those with respect to curved coordinates $r$, we need the relations between positions, velocities, and accelerations in curved coordinates and in joint space. We let the position of the end-effector be described as follows:

$$
\begin{aligned}
r & \in R^{n}, \\
r & =\left[r_{n}^{T}, r_{t}^{T}\right]^{T} \\
r_{n} & =\left[\phi_{1}(x), \ldots, \phi_{m}(x)\right]^{T}=\Phi(x) \in R^{m} \\
r_{t} & =\left[\psi_{1}(x), \ldots, \psi_{n-m}(x)\right]^{T}=\Psi(x) \in R^{n-m}
\end{aligned}
$$

and

$$
q^{T}=\left[q_{r}^{T}, q_{f}^{T}\right]^{T}
$$

where $r_{n}$ and $r_{t}$ denote the normal part and the tangential part of the coordinate, respectively. Then,

$$
\begin{aligned}
& \dot{r}=J_{r q_{r}} \dot{q}_{r}+J_{r q_{f}} \dot{q}_{f} \\
& \ddot{r}=J_{r q_{r}} \ddot{q}_{r}+J_{r q_{f}} \ddot{q}_{f}+\dot{J}_{r q_{r}} \dot{q}_{r}+\dot{J}_{r q_{f}} \dot{q}_{f}
\end{aligned}
$$

and

$$
\left[\begin{array}{c}
\tau_{t r} \\
\tau_{t f}
\end{array}\right]=\left[\begin{array}{c}
J_{r q_{r}}^{T} \\
J_{r q_{\ell}}^{T}
\end{array}\right] f_{t}
$$

where $J_{r q_{r}}=\frac{\partial r}{\partial q_{r}}, J_{r q_{f}}=\frac{\partial r}{\partial q_{f}}$, and $f_{t} \in R^{n-m}$ is the tangential cutting force in curved coordinates. Assume that the flexible manipulator is non-redundant with respect to the rigid part so that $J_{r a_{r}}$ is insured to be invertible. As a result, we can obtain the dynamic equations in the curved coordinates as follows:

$$
\begin{aligned}
\ddot{r}= & N_{1}\left(q_{r}, q_{f}, \dot{q}_{r}, \dot{q}_{f}\right) \dot{r}+N_{2}\left(q_{r}, q_{f}, \dot{q}_{r}, \dot{q}_{f}\right) \dot{q}_{f} \\
& +N_{3}\left(q_{r}, q_{f}\right)+N_{4}\left(q_{r}, q_{f}\right) K q_{f}+N_{5}\left(q_{r}, q_{f}\right) \tau \\
& +N_{6}\left(q_{r}, q_{f}\right)
\end{aligned}
$$

And the flexible part can be expressed in a more compact form as follows:

$$
\begin{aligned}
\ddot{q}_{f}= & B_{1}\left(q_{r}, q_{f}, \dot{q}_{r}, \dot{q}_{f}\right) \dot{r}+B_{2}\left(q_{r}, q_{f}, \dot{q}_{r}, \dot{q}_{f}\right) \dot{q}_{f} \\
& +B_{3}\left(q_{r}, q_{f}\right)+B_{4}\left(q_{r}, q_{f}\right) K q_{f}+B_{5}\left(q_{r}, q_{f}\right) \tau \\
& +B_{6}\left(q_{r}, q_{f}\right)
\end{aligned}
$$


For solving the vibration problem, we will apply the singular perturbation theory here and decompose the flexible manipulator system into a slow subsystem and a fast subsystem. To that aim, we make the some definitions as follows:

$$
\begin{gathered}
\nu=K q_{f} \quad \text { and } \quad \tilde{K}=K \epsilon^{2}, \\
z_{1}=\nu \quad \text { and } \quad z_{2}=\epsilon \dot{\nu} \\
y_{1}=r \quad \text { and } \quad y_{2}=\dot{r}
\end{gathered}
$$

where $\epsilon^{2}$ is a common factor extracted from each entry of the matrix $K$, assumed to be small enough. Hence, acccording to (11) and (12), the state space form of a singularly perturbed model can thus be derived as follows:

$$
\begin{aligned}
\dot{y}_{1}= & y_{2} \\
\dot{y_{2}}= & N_{1} y_{2}+\epsilon N_{2} \tilde{K}^{-1} z_{2}+N_{3}+N_{4} z_{1} \\
& +N_{5} \tau+N_{6} \\
\epsilon \dot{z}_{1}= & z_{2} \\
\epsilon \dot{z}_{2}= & \tilde{K}\left(B_{1} y_{2}+\epsilon B_{2} \tilde{K}^{-1} z_{2}+B_{3}+B_{4} z_{1}\right. \\
& \left.+B_{5} \tau+B_{6}\right)
\end{aligned}
$$

which amounts to the singular perturbation model of the flexible manipulator system. It is obvious that if the stiffness matrix $K$ is very large or, equivalently, $\epsilon$ is very small, the flexible manipulator system can be treated like a rigid manipulator system. For the extreme case where $\epsilon \rightarrow 0$, we can obtain the following relation via (14):

$$
\begin{aligned}
& \bar{z}_{2}=0 \\
& \bar{z}_{1}=-\bar{B}_{4}^{-1}\left[\bar{B}_{1} \bar{y}_{2}+\bar{B}_{3}+\bar{B}_{5} \bar{\tau}+\bar{B}_{6}\right],
\end{aligned}
$$

By substituting it into (13) with $\epsilon=0$, we can obtain the following set of equations:

$$
\begin{aligned}
\dot{\bar{y}}_{1}= & \bar{y}_{2} \\
\dot{\bar{y}}_{2}= & \bar{N}_{1} \bar{y}_{2}+\bar{N}_{3}+\bar{N}_{4}\left[-\bar{B}_{4}^{-1}\left(\bar{B}_{1} \bar{y}_{2}+\bar{B}_{3}+\bar{B}_{5} \tau+\bar{B}_{6}\right)\right] \\
& +\bar{N}_{5} \tau+\bar{N}_{6} \\
= & {\left[\overline{\bar{J}}_{r}-\bar{J}_{r} \bar{M}_{r r}^{-1} \bar{C}_{r r}\right] \bar{J}_{r}^{-1} \bar{y}_{2}-\bar{J}_{r} \bar{M}_{r r}^{-1} \bar{G}_{1}+\bar{J}_{r} \bar{M}_{r r}^{-1} \bar{\tau} } \\
& +\bar{J}_{r} \bar{M}_{r r}^{-1}\left(\bar{A}_{1}^{T} \bar{\lambda}+\bar{J}_{r}^{T} \bar{f}_{t}\right)
\end{aligned}
$$

where all the variables and functions with overbar are used to denote those in (13), (14) in the situation when $\epsilon=0$. This system will then be referred to as the slow subsystem.

Then, we rewrite the state-space equation (16) into a set of differential equations, by premuliplying the equations by $\bar{J}_{r q_{r}}^{-T} \bar{M}_{r r} \bar{J}_{r q_{r}}^{-1}$. Then the resulting equations become:

$$
\begin{aligned}
\bar{J}_{r q_{r}}^{-T} \bar{M}_{r r} \bar{J}_{r q_{r}}^{-1} \ddot{\bar{r}}+ & {\left[\bar{J}_{r q_{r}}^{-T} \bar{C}_{r r} \bar{J}_{r q_{r}}^{-1}-\bar{J}_{r q_{r}}^{-T} \bar{M}_{r r} \bar{J}_{r q_{r}}^{-1} \dot{\bar{J}}_{r q_{r}} \bar{J}_{r q_{r}}^{-1}\right] \dot{\bar{r}} } \\
& +\bar{J}_{r q_{r}}^{-T} \bar{G}_{1}=\bar{J}_{r q_{r}}^{-T} \tau+\bar{J}_{r q_{r}}^{-T} \bar{A}_{1}^{T} \lambda+\bar{f}_{t} \quad(17)
\end{aligned}
$$

where $\bar{f}=\bar{J}_{r}^{-T} \bar{\tau}$. For convenience of analysis, we can further rewrite the above equations into the following form:

$$
\bar{M}(\bar{r}) \ddot{\bar{r}}+\bar{C}(\bar{r}, \dot{\bar{r}}) \dot{\bar{r}}+\bar{G}(\bar{r})=\bar{f}+\bar{K} \bar{\lambda}+\bar{f}_{t}
$$

$$
\begin{gathered}
{\left[\begin{array}{cc}
\bar{M}_{11} & \bar{M}_{12} \\
\bar{M}_{21} & \bar{M}_{22}
\end{array}\right]\left[\begin{array}{c}
\ddot{\bar{r}}_{n} \\
\ddot{\bar{r}}_{t}
\end{array}\right]+\left[\begin{array}{cc}
\bar{C}_{11} & \bar{C}_{12} \\
\bar{C}_{21} & \bar{C}_{22}
\end{array}\right]\left[\begin{array}{c}
\dot{\bar{r}}_{n} \\
\dot{\bar{r}}_{t}
\end{array}\right]+\left[\begin{array}{c}
\bar{G}_{11} \\
\bar{G}_{21}
\end{array}\right]} \\
=\left[\begin{array}{c}
\bar{f}_{m n} \\
\bar{f}_{m t}
\end{array}\right]+\left[\begin{array}{c}
I_{m} \\
0
\end{array}\right] \bar{\lambda}+\left[\begin{array}{c}
0 \\
\bar{f}_{t}
\end{array}\right]
\end{gathered}
$$

where

$$
\begin{aligned}
{\left[\begin{array}{ll}
\bar{M}_{11} & \bar{M}_{12} \\
\bar{M}_{21} & \bar{M}_{22}
\end{array}\right] } & =\bar{J}_{r q_{r}}^{-T} \bar{M}_{r r} \bar{J}_{r q_{r}}^{-1} \\
{\left[\begin{array}{ll}
\bar{C}_{11} & \bar{C}_{12} \\
\bar{C}_{21} & \bar{C}_{22}
\end{array}\right] } & =\bar{J}_{r q_{r}}^{-T} \bar{C}_{r r} \bar{J}_{r q_{r}}^{-1}-\bar{J}_{r q_{r}}^{-T} \bar{M}_{r r} \bar{J}_{r q_{r}}^{-1} \dot{J}_{r q_{r}} \bar{J}_{r q_{r}}^{-1} \\
{\left[\begin{array}{l}
\bar{G}_{11} \\
\bar{G}_{21}
\end{array}\right] } & =\bar{J}_{r q_{r}}^{-T} \bar{G}_{1}
\end{aligned}
$$

and

$$
\bar{K}=\left[\begin{array}{c}
I_{m} \\
0
\end{array}\right]
$$

Since we assume that the end-effector traverses along the constrained curved surface, it should be intuitive to say that $\bar{r}_{n}=\dot{\bar{r}}_{n}=\ddot{\vec{r}}_{n}=0$. Thus, from (19) the following equations can be obtained.

$$
\begin{aligned}
& \bar{M}_{12} \ddot{\bar{r}}_{t}+\bar{C}_{12} \dot{\bar{r}}_{t}+\bar{G}_{11}=\bar{f}_{m n}+\bar{\lambda} \\
& \bar{M}_{22} \ddot{\bar{r}}_{t}+\bar{C}_{22} \dot{\bar{r}}_{t}+\bar{G}_{21}=\bar{f}_{m t}+\bar{f}_{t} .
\end{aligned}
$$

To derive the fast subsystem, we first define the fast time-scale as $\mu=\frac{t}{\epsilon}$ and then redefine the fast variables as

$$
\begin{aligned}
& \eta_{1}=z_{1}-\bar{z}_{1} \\
& \eta_{2}=z_{2}-\bar{z}_{2}
\end{aligned}
$$

As $\epsilon \rightarrow 0$, we can obtain $\frac{d y_{1}}{d \mu}=\frac{d y_{2}}{d \mu}=0$, which implies that $y_{1}$ and $y_{2}$ are constants with respect to the fast time-scale $\mu$, i.e., within the boundary layer, $y_{1}$ and $y_{2}$ are stationary. Therefore, the fast subsystem can be easily derived as

$$
\begin{aligned}
& \frac{d \eta_{1}}{d \mu}=\eta_{2} \\
& \frac{d \eta_{2}}{d \mu}=-\tilde{K} \bar{H}_{22} \eta_{1}+\tilde{K} \bar{H}_{21}(\tau-\bar{\tau})
\end{aligned}
$$

Similarly, we can also refer the equations (20) and (21) as force part and motion part, respectively. According to these two subsystems, slow subsystem and fast subsystem, an adaptive composite controller will be proposed in the next chapter.

\section{CONTROLLER DESIGN}

\section{Slow Subsystem Controller}

There are some useful properties of the slow subsystem to design the slow subsystem controller.

\section{Proposition 3.1 :}

$\bar{M}_{22}$ in (21) is symmetric and positive definite.

Proposition 3.2 : 
By a proper choice of $C(q, \dot{q})$ to define $\bar{C}_{22}$ in (21), the matrix $\dot{M}_{22}-2 \bar{C}_{22}$ is skew-symmetric.

\section{Proposition 3.3 :}

There exist some constant system parameter vectors $\bar{\theta}_{1}$ and $\bar{\theta}_{2}$ such that

$$
\begin{aligned}
& \bar{M}_{12} \dot{u}+\bar{C}_{12} u-\bar{G}_{11}=\bar{w}_{1}^{T} \bar{\theta}_{1} \\
& \bar{M}_{22} \dot{u}+\bar{C}_{22} u-\bar{G}_{21}=\bar{w}_{2}^{T} \bar{\theta}_{2}
\end{aligned}
$$

where $u$ is a smooth variable with proper definition and $\bar{w}_{1}$ and $\bar{w}_{2}$ are known function matrices.

In the following, the design procedure for controller for slow subsystem will be described. We define an error signal as

$$
\tilde{\tilde{r}}_{t}=\bar{r}_{t}-r_{t d}
$$

Then, we define an auxiliary signal $\bar{s}$ as

$$
\bar{s}=\dot{\bar{r}}_{t}+K_{r} \tilde{\bar{r}}_{t}
$$

where $K_{r}$ is some positive constant, $r_{t d}$ is the desired positional trajectory in tangential direction. According to Proposition 3.1, 3.2 and 3.3, the error dynamics of equation (21) can be derived as follows :

$$
\begin{aligned}
\bar{M}_{22} \dot{\bar{s}}+\bar{C}_{22} \bar{s}= & \bar{M}_{22} \ddot{\bar{r}}_{t}+\bar{C}_{22} \dot{\bar{r}}_{t}+\bar{G}_{21} \\
& +\bar{M}_{22}\left(-\ddot{r}_{t d}+K_{r}\left(\dot{\bar{r}}_{t}-\dot{r}_{t d}\right)\right) \\
& +\bar{C}_{22}\left(-\dot{r}_{t d}+K_{r}\left(\bar{r}_{t}-r_{t d}\right)\right)-\bar{G}_{21} \\
= & \bar{f}_{m t}+\bar{f}_{t}+\ddot{w}_{2}^{T} \bar{\theta}_{2}
\end{aligned}
$$

Then, we can design the controller $\bar{f}_{m t}$ as :

$$
\bar{f}_{m t}=-\bar{w}_{2}^{T} \hat{\bar{\theta}}_{2}-\bar{f}_{t}-K_{p} \bar{s}
$$

and parameter adaptation law as:

$$
\dot{\overline{\theta_{2}}}=\Gamma_{2}^{-1} \bar{w}_{2} \bar{s}
$$

Theorem 3.1: $\quad$ Consider the slow subsystem (20)(21) with the control law (28) and the adaptation law (29). Then, the tracking errors in position will converge to zero.

Proof: Let the Lyapunov function candidate $\bar{V}_{11}$ as:

$$
\bar{V}_{11}=\frac{1}{2} \bar{s}^{T} \bar{M}_{22} \bar{s}+\frac{1}{2} \tilde{\bar{\theta}}_{2}^{T} \Gamma_{2} \tilde{\bar{\theta}}_{2}
$$

and then take its time derivative along the trajectories of (27) to obtain

$$
\begin{aligned}
\frac{d}{d t} \bar{V}_{11} & =\bar{s}^{T} \bar{M}_{22} \dot{\bar{s}}+\frac{1}{2} \bar{s}^{T} \dot{\bar{M}}_{22} \bar{s}+\dot{\bar{\theta}}_{2}^{T} \Gamma_{2} \tilde{\hat{\theta}}_{2} \\
& =\bar{s}^{T} \bar{M}_{22} \dot{\bar{s}}+\bar{s}^{T} \bar{C}_{22} \bar{s}-\dot{\hat{\theta}}_{2}^{T} \Gamma_{2} \tilde{\bar{\theta}}_{2} \\
& =\bar{s}^{T}\left(\bar{w}_{2}^{T} \tilde{\tilde{\theta}}_{2}-K_{p} \bar{s}\right)-\bar{s}^{T} \bar{w}_{2}^{T} \overline{\tilde{\theta}}_{2} \\
& =-\bar{s}^{T} K_{p} \bar{s} \\
& \leq-\gamma_{1}\|\bar{s}\|^{2} \\
& \leq 0
\end{aligned}
$$

where $\tilde{\bar{\theta}}_{2}=\vec{\theta}_{2}-\hat{\bar{\theta}}_{2}, \gamma_{1}$ is positive constant.
Therefore, we can guarantee that $\tilde{\bar{\theta}}_{2} \in \mathcal{L}_{\infty}, \bar{s} \in \mathcal{L}_{\in} \cap$ $\mathcal{L}_{\infty}$ and hence $\dot{\bar{s}} \in \mathcal{L}_{\infty}$. By applying Barbalat's lemma, we can obtain that $\bar{s} \rightarrow 0$ when $t \rightarrow \infty$, and so will $\tilde{\tilde{r}}_{t}, \dot{\tilde{r}}_{t}$.

Furthermore, for designing $\vec{f}_{m n}$ to force $\bar{\lambda}$ to approach the desired contact normal force, called $\lambda_{d}$, we derive the error dynamics as follows :

$$
\begin{aligned}
\bar{M}_{12} \dot{\bar{s}}+\bar{C}_{12} \bar{s}= & \bar{M}_{12} \ddot{\bar{r}}_{t}+\bar{C}_{12} \dot{\bar{r}}_{t}+\bar{G}_{11} \\
& +\bar{M}_{12}\left(-\ddot{r}_{t d}+K_{r}\left(\dot{\bar{r}}_{t}-\dot{r}_{t d}\right)\right) \\
& +\bar{C}_{12}\left(-\dot{r}_{t d}+K_{r}\left(\bar{r}_{t}-r_{t d}\right)\right)-\bar{G}_{11} \\
= & \bar{f}_{m n}+\bar{\lambda}+\bar{w}_{1}^{T} \bar{\theta}_{1}
\end{aligned}
$$

Let control law $\bar{f}_{m n}$ be designed as:

$$
\bar{f}_{m n}=-\bar{w}_{1}^{T} \hat{\bar{\theta}}_{1}-\lambda_{d}
$$

Thus, the error dynamics become:

$$
\begin{aligned}
\bar{M}_{12} \dot{\bar{s}}+\bar{C}_{12} \bar{s} & =\bar{w}_{1}^{T} \tilde{\bar{\theta}}_{1}-\left(\lambda_{d}-\bar{\lambda}\right) \\
& =\bar{w}_{1}^{T} \tilde{\bar{\theta}}_{1}-\bar{e}_{f}
\end{aligned}
$$

where $\bar{e}_{f}=\lambda_{d}-\bar{\lambda}$. Since $\dot{\bar{s}} \rightarrow 0$ as $t \rightarrow \infty$, for sufficent large $t(>N$, a large positive interger $)$, we can get

$$
\bar{w}_{1}^{T} \overline{\bar{\theta}}_{1}-\bar{e}_{f} \approx 0
$$

Furthermore, adaptive law $\dot{\overline{\hat{\theta}}}_{1}$ is designed as:

$$
\dot{\hat{\theta}}_{1}=\Gamma_{1}^{-1} \bar{w}_{1} \bar{e}_{f}
$$

Theorem 3.2: $\quad$ Consider the slow subsystem (20)(21) with the control law (28)(33) and the adaptation law (29)(36). Then, the force errors will converge to zero as $t \rightarrow \infty$.

Proof: Consider the Lyapunov function $\bar{V}_{12}=\frac{1}{2} \tilde{\bar{\theta}}_{1}^{T} \tilde{\bar{\theta}}_{1}$ and take its time derivative as:

$$
\begin{aligned}
\dot{\bar{V}}_{12} & =\tilde{\bar{\theta}}_{1}^{T} \dot{\bar{\theta}}_{1} \\
& =\tilde{\bar{\theta}}_{1}^{T}\left(-\Gamma_{1}^{-1} \tilde{w}_{1} \bar{e}_{f}\right) \\
& =\tilde{\bar{\theta}}_{1}^{T}\left(-\Gamma_{1}^{-1} \bar{w}_{1} \bar{w}_{1}^{T}\right) \tilde{\bar{\theta}}_{1} \\
& \leq 0
\end{aligned}
$$

Therefore, if $\bar{w}_{1}$ satisfies the persistent excitation(P.E.) condition, $\tilde{\bar{\theta}}_{1}$ will approach zero as $t \rightarrow \infty$. Hence, the force error $\bar{e}_{f}$ will also converge to zero as $t \rightarrow \infty$.

Remark1: To assure that $\bar{w}_{1}$ is a persistently exciting vector, we modify the original desired trajectory $\bar{r}_{t d}$ by adding a small persistently exciting term, i.e., $\bar{r}_{t d}^{\prime}=\bar{r}_{t d}+\alpha \bar{w}_{p e}$, where $\bar{r}_{t d}^{\prime}$ is the new desired trajectory and $\bar{w}_{p e}$ is a small persistently exciting term. Thus, $\bar{w}_{1} \in P . E$., then $\tilde{\bar{\theta}}_{1} \rightarrow 0$ as $t \rightarrow \infty$, resulting in $\bar{e}_{f} \rightarrow 0$ as $t \rightarrow \infty$.

Remark2: In practice, implementation of the adaptation law (29)(36) will usually incorporate the so-called $\sigma$-modification term, i.e.,

$$
\begin{aligned}
& \dot{\hat{\bar{\theta}}}_{2}=\Gamma_{2}^{-1}\left(\bar{w}_{2} \bar{s}-\sigma \hat{\bar{\theta}}_{2}\right) \\
& \dot{\hat{\hat{\theta}}}_{1}=\Gamma_{1}^{-1}\left(\bar{w}_{1} \bar{e}_{f}-\sigma \hat{\bar{\theta}}_{1}\right)
\end{aligned}
$$


in order to robustify the system relative to some nonideal terms.

\section{Fast Subsystem Controller}

The fast subsystem (22) is rewritten in a very compact form as follows :

$$
\frac{d \eta}{d \mu}=\bar{A} \eta+\bar{B} \tau_{f}
$$

where

$$
\begin{aligned}
\eta & =\left[\begin{array}{ll}
\eta_{1}^{T} & \eta_{2}^{T}
\end{array}\right], \quad \bar{A}=\left[\begin{array}{cc}
0 & I \\
-\tilde{K} \bar{H}_{22} & 0
\end{array}\right], \quad \bar{B}=\left[\begin{array}{c}
0 \\
\tilde{K} \bar{H}_{21}
\end{array}\right], \\
\tau_{f} & =\tau-\bar{\tau} .
\end{aligned}
$$

Our control objective here is clearly to supress the vibrations of the flexible links, i.e., to force $\eta \rightarrow 0$ and, hence, a robust regulator will serve our need. Within the boundary layer, the system matrices $\bar{A}$ and $\bar{B}$ can be replaced by $\bar{A}_{0}+\Delta \bar{A}_{0}$ and $\bar{B}_{0}+\Delta \bar{B}_{0}$, respectively, where $\bar{A}_{0}$ and $\bar{B}_{0}$ are nominal matrices with known elements and there are availabe known bounds on $\left\|\Delta \bar{A}_{0}\right\|$ and $\left\|\Delta \bar{B}_{0}\right\|$.

Here, after a careful study, a proper design of the aforementioned regulator is apparently a dynamic feedback controller expressed as follows :

$$
\frac{d \tau_{f}}{d \mu}=\bar{F} \eta+\bar{G} \tau_{f}
$$

where the matrices $\bar{F}$ and $\bar{G}$ will be determined later. By adopting such control, the closed-loop fast subsystem becomes

$$
\frac{d \zeta}{d \mu}=\mathcal{A} \zeta+\mathcal{B} \zeta
$$

where $\zeta=\left[\eta^{T}, \tau_{f}^{T}\right]^{T}, \mathcal{A}=\left[\begin{array}{cc}\bar{A}_{0} & \bar{B}_{0} \\ \bar{F} & \bar{G}\end{array}\right]$, and $\mathcal{B}=$ $\left[\begin{array}{cc}\Delta \bar{A}_{0} & \Delta \bar{B}_{0} \\ 0 & 0\end{array}\right]$. The following theorem summarizes a condition under which the above design of the fast subsystem controller may provide the desirable result. Theorem 3.3: If $\bar{F}$ and $\bar{G}$ are chosen such that $\mathcal{A}$ is Hurwitz and there exist $P, Q>0$ satisfying

$$
\mathcal{A}^{T} P+P \mathcal{A}=-Q
$$

and $\lambda_{\min }(Q)>2 \alpha\|P\|$, where $\|\mathcal{B}\| \leq \alpha$, then it is guaranteed that $\|\zeta\| \rightarrow 0$ exponentially.

Proof: The proof is omitted here due to limited space. One can see [28] for details.

\section{Composite Controller:}

Here, the proposed composite controller is the combination of the slow subsystem controller $\bar{\tau}$ and the fast subsystem controller $\tau_{f}$, i.e.,

$$
\tau=\bar{\tau}+\tau_{f}
$$

The control results of such system are detailedly summarized in the following theorem.

Theorem 3.4: Consider the system (11) and (12) with the composite control law (28), (33), (39) and the adaptation law (29), (36). Then, both tracking errors in position and contact force and link vibration will converge to zero as $t \rightarrow \infty$.

Proof: The proof is omitted here due to limited space. One can see [28] for details.

Remark3: If the adaptation law has incorporated the $\sigma$-modification as stated in Remark2 $\left(\dot{\hat{\theta}}_{2}=\Gamma_{2}^{-1}\left(w_{2} s-\right.\right.$ $\left.\left.\sigma \hat{\theta}_{2}\right), \dot{\hat{\theta}}_{1}=\Gamma_{1}^{-1}\left(w_{1} e_{f}-\sigma \hat{\theta}_{1}\right)\right)$, then the error convergence will have to be modified so that they only converge to a residual set whose size depends on the $\sigma$ modification parameter.

\section{SIMULATION AND EXPERIMENTAL RESULTS}

A 2-link planar flexible manipulator deburring system has been set up and experimented in Department of Electrical Engineering at National Taiwan University (NTU). The 2-link flexible manipulator is drived by two revolute joints which are perpendicular to the motional plane. The first link is driven by a D.C. motor with ratio $128: 1$, and the second link is driven by a D.C. Brushless motor with gear ratio 100:1. The grinding tool is equipped at the tip of the second link. In the experiment, the flexible modes are measured using strain gauges. A PC 486-33 is used as the processor to implement the computation of the control law and the adaptation law, in which the sampling rate is set to be $300 \mathrm{~Hz}$. The workpiece chosen for deburring is a rectagulat steer slip, which is held paralel to the $x_{2}$-axis direction int the task space.

A computer simulation results of a case of a 2 -link plannar flexible maipulator deburring system will be shown to verify the performance of the previous design of the composite controller. The constraint surface of the workpiece can be mathematically represented as the following equations:

$$
r=\left[r_{n}^{T}, r_{t}^{T}\right]^{T}
$$

where $r_{n}=x_{1}-L$ and $r_{t}=x_{2}, x_{1}, x_{2}$ are the coordinates of Cartesian space, and in this case, $L$ is chosen as $0.85 \mathrm{~m}$.

Assume that the gravitational force and the torsion effect of link can be neglected. Here, we use 2 modes to describe each link's deformation. The desired contact normal force and desired chamfering force are chosen as $\lambda_{d}=2.0 \mathrm{nt}$ and $f_{t d}=0.5 \mathrm{nt}$. Fig. 3 (a) (b) show the normal cutting force and tangential postional tracking trajectories, respectively, (c) shows the tangential cutting force, (d) and (e) show the input torque, and (f) shows the joint angles. From these figures of the simulation results we know that the tracking errors converge and all system states and control inputs are bounded 

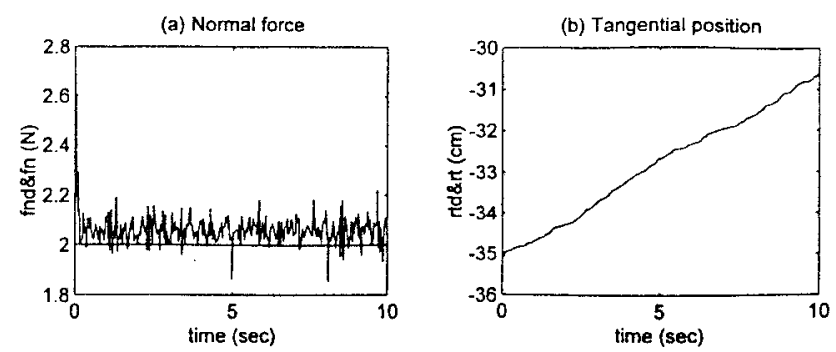

(c) Tangential force
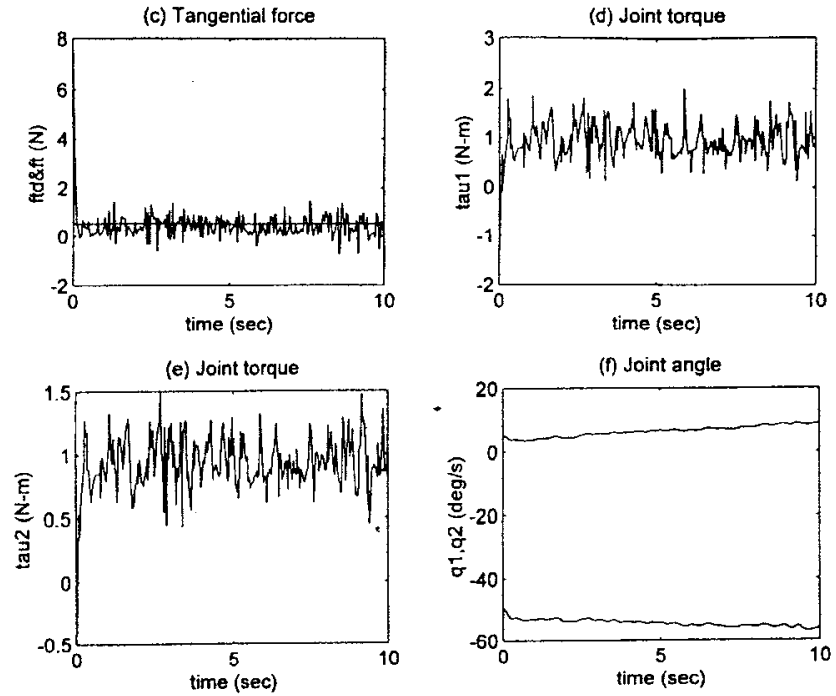

Figure 3: Simulation result

simutaneously. Therefore, the effectiveness of the composite controller is verified.

In the experiment case, the constraint surface of the workpiece can be mathematically represented as $x_{1}=0.96$. The desired contact normal force and desired chamfering force are chosen as $\lambda_{d}=1.0 \mathrm{nt}$ and $f_{t d}=0.2$ nt. Fig. 4 (a) (b) show the normal cutting force and tangential postional tracking trajectories , respectively, (c) shows the tangential cutting force, (d) and (e) show the input torque, and (f) shows the joint angles. From these figures of the experimental results, the preformance of the composite controller is verified.

Note: In order to robustify the system relative to some nonideal terms, the adaptation law in simulation and experiment has incorporated the $\sigma$-modification as stated in Remark2.

\section{CONCLUSION}

In this paper, we derived a new dynamic model of a deburring flexible manipulator under the curved coordinates. A new controller was proposed for maintaining a constant contact normal force and maintaining a desired constant tangential cutting force. To satisfy this two requirements, we adopted adaptive hybrid force/position control strategy for flexible deburring manipulator. The dynamic model of a $n$-link deburring flexible manipulator was derived using the Lagrangian
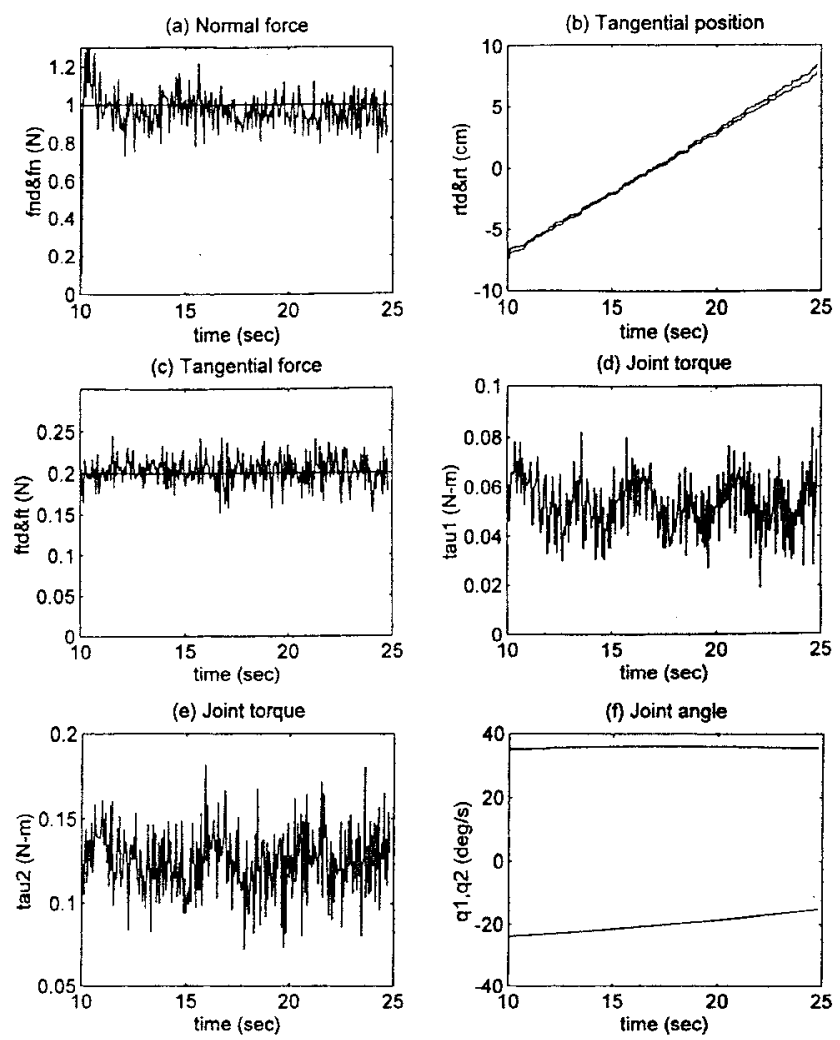

Figure 4: Experimental result

method with the assumed-mode approach. The singular perturbation method was utilized to formulate the model into a two time-scale system. Hence, we can design the controller for slow subsystem and fast subsystem, respectively. For our deburring requirements, we use the adaptive hybrid force/position controller to control the slow subsystem, and the fast subsystem is controlled by a dynamic feedback controller. According to our controller design, tracking errors of position and force as well as the link vibrations can be shown to converge to zero as time approach infinity. For demonstrating the effectiveness of the controller, some computer simulations and experiments were performed.

\section{References}

[1] Lian, F.-L., J.-H. Yang and L.-C. Fu, "Adaptive hybrid position/force control for flexible mnipulators in task space," Proc. National Symposium on Automatic Control, pp. 210-214, 1995.

[2] Kazerooni, H., J. J. Bausch and B. B. Kramer, "An approach to automated deburring by robot manipulators," ASME J. Dyna. Syst. Meas. Contr., vol. 108, pp. 354-359, Dec. 1986.

[3] Bin Yao and Masayoshi Tomizuka, "Adaptive control of robot manipulators in constrained motion controller design, " ASME J. Dyna. Syst. Meas. Contr., vol. 117, pp320-328, Sep.1995.

[4] Robert R. Y. Zhen and Andrew A. Goldenberg, "Variable structure hybrid control of manipulators 
in unconstrained and constrained motion," $A S M E$ J. Dyna. Syst. Meas. Contr., vol.118, pp327-332, June 1996.

[5] Liu, S. and H. Asada, "Transferring manipulative skills to robots: representation and acquisition of tool manipulative skills using a process dynamics model, " ASME J. Dyna. Syst. Meas. Contr., vol. 114, pp. 220-228, June 1992.

[6] Bone G. M., M. A. Elbestawi, R. Lingarkar and L. Liu, "Force control for robotic deburring, " ASME J. Dyna. Syst. Meas. Contr., vol. 113, pp. 395-400, Sep. 1991.

[7] Liu, S. and H. Asada, "Teaching and learning of deburring robots using neutal networks, " Proc. of 1993 IEEE, pp. 339-345, 1993.

[8] Her, M. G. and H. Kazerooni, "Automated robotic deburring of parts using compliance control," ASME J. Dyna. Syst. Meas. Contr., vol. 113, pp. 60-66, Mar. 1991.

[9] Kazerooni, H., "Direct-drive active compliant end effector (Active RCC), "IEEE J. Robotics Automat., vol. 4, no. 3, pp. 324-333, June 1988.

[10] Jeon, D. and M. Tomizuka, "Learning hybrid force and position control of robot manipulators," IEEE Trans. Robot. Automat., vol. 9, no. 4, pp. 423-431, Aug. 1993.

[11] Lian, F.-L., "Nonlinear adaptive control for constrained flexible manipulator," Master Thesis, Institute of Electrical Engineering, National Taiwan University, Taiwan, R.O.C., 1994.

[12] Luca, D. A. and B. Siciliano, "Closed-form dynamic model of planar multilink lightweight robots," IEEE Trans. Syst. Man Cybernetics, vol. 21, no. 4, pp. 826-839, July/August 1991.

[13] Matsuno, F., T. Asano and Y. Sakawa, "Modeling and quasi-static hybrid position/force control of constrained planar two-link flexible manipulators, " IEEE Trans. Robotics Automation, vol. 10, no. 3, pp. 287-297, June 1994.

[14] McClamroch, N. H. and D. Wang, "Feedback stabilization and tracking of constrained robots," IEEE Trans. Automat. Contr., vol. 33, no. 5, pp. 419-426, May. 1988.

[15] Siciliano, B. and W. J. Book, "A singular perturbation approach to control of lightweight flexible manipulators, "Int. J. Robotics Res., vol. 7, no. 4, pp. 79-90, Aug. 1988.

[16] Lewis, F. L., C. T. Abdallah and D. M. Dawson, "Control of Robot Mnipulators," Macmillan, pp. 340-346, 1993.

[17] Raibert, M. H. and Craig, J. J., "Hybrid position/force control of manipulators, "ASME J. Dyna. Syst. Meas. Contr., vol. 102, pp. 126-133. 1981.

[18] Yang, J.-H., "Nonlinear adaptive control for manipulators with flexible components : constrained and unconstrained cases," Doctor Thesis, Institute of Electrical Engineering, National Taiwan University, Taiwan, R.O.C., 1995.

[19] Slotine, J.-J. E. and W. Li, "Adaptive manipulator control : a case study," IEEE Trans. Automat.
Contr., vol. 33, no. 11, pp995-1003, 1988.

[20] Jean, J.-H. and L.-C. Fu, "Adaptive hybrid control strategies for constrained robots, "IEEE trans. Robot. Automat., vol. 38, no. 4, pp. 598-603, April 1993.

[21] Yang, J.-H., F.-C. Liu and L.-C. Fu, "Nonlinear control for flexible manipulator," Proc. IEEE American Cntr. Conf., pp. 2873-2877, 1933.

[22] Matsuno F., T. Asano and Y. Sakawa, "Modeling and quasi-static hybrid position/force control of constrained planar two-link flexible manipulator," IEEE Trans. Robot. Automat., vol. 10, no. 3., pp. 287-168, June 1994.

[23] H. Kazerooni, "On the robot compliant motion control," ASME J. Dyna. Syst. Meas. Contr., vol. 111, pp. 416-425, Sep. 1989.

[24] Bone G. M. and M. A. Elbestawi, "Robotic force for deburring using an active end effector, "Robotica, vol. 7, pp. 303-308, 1989.

[25] Liu, S. and Ken-ichiro Shimokura, "Programming deburring robots based on human demonstration with direct burr size measurement" Proc. IEEE Robot. and Automation, pp. 572-577, 1994.

[26] Bone G. M. and M. A. Elbestawi, "Sensing and control for automated robotic edge deburring, " IEEE Trans. Robot. Automat., vol. 41, no. 2., pp. 137-146, April 1994.

[27] Yao, B., Chan, S. P., and Wang, D., "A Unified Approach to Variable Structure Control of Robot Manipulators, " Proc. of American Control Conference, pp. 1282-1286, 1992.

[28] Lin, I.-C, "Adaptive hybrid force/position control of a flexible manipulator for automated deburring with on-line cutting trajectory modification," Master Thesis, Institute of Electrical Engineering, $\mathrm{Na}$ tional Taiwan University, Taiwan, R.O.C., 1996. 